\title{
WHAT WE CAN SAY ABOUT PN IF THEIR LUMINOSITY FUNCTION DISTANCES ARE CORRECT
}

\author{
GEORGE H. JACOBY \\ National Optical Astronomy Observatories \\ P.O. Box 26732, Tucson, AZ 85726
}

\begin{abstract}
I review the arguments favoring the high reliability of the PNLF method. Agreement with Cepheid distances is better than 8\%, and consistency for multiple PNLF distances within clusters is even better. Agreement between Cepheid distances to spirals and PNLF distances to ellipticals within the same cluster also is excellent. In order for the PNLF method to work despite the vast diversity of properties seen among PN, several factors must operate. Most importantly, the progenitors of the bright extragalactic PN probably have ages less than $\sim 10$ Gyrs. Also, very young PN must either be absent from the bright extragalactic samples or must have lower luminosities than suggested by their predicted central star masses. The latter may be due to internal dust, nitrogen enrichment that competes with oxygen for ionizing photons, and/or a tendency for massive PN to be optically thin to ionizing radiation. In addition, models that reproduce observed PNLFs rely on theoretical evolutionary tracks of central stars, and so these tracks also must be reasonably correct. PNLF observations suggest, however, that the slope of the initial-to-final mass relation is shallower than the Weidemann (1987) relation.
\end{abstract}

\section{Introduction}

Planetary Nebulae (PN) exhibit tremendous diversity in their characteristics. Their morphology (bipolar, elliptical, multiple shells) is highly varied, and some PN contain considerable dust. PN are formed from a wide range of progenitor ages (a few Myr to many Gyr), masses ( 0.8 to $8 \mathrm{M}_{\odot}$ ), and metallicities $\left(0.01\right.$ to $\left.2 \mathrm{Z}_{\odot}\right)$. Also, $\mathrm{PN}$ have luminosities that extend more than 8 mags. 


\section{PNLF DISTANCES TO GALAXIES}

A simple argument, though, suggests that PN diversity is not overly important to the planetary nebula luminosity function (PNLF) technique. That is, if all PN have similar central stars characterized solely by their core masses, then all PN have a similar maximum number of ionizing photons to power their nebulae. That maximum occurs at a time when the central star is very hot, but has not yet begun to decline in luminosity. Consequently, if the nebulae absorb most or all of the ionizing radiation, the nebulae will transform that power into emission lines, with the [OIII] $\lambda 5007$ line emitting up to $15 \%$ of the incident ionizing flux. Furthermore, the time history of the central star is determined by its mass so that time histories of the [OIII] emission will be similar. Thus, if all central stars are similar, then there will be a similar maximum [OIII] flux and a repeatable flux history and luminosity function (PNLF) for all populations.

Despite variations in their details (e.g., geometry, metallicity, size, age), large ensembles of PN will have similar maximum luminosities. If this assertion can be demonstrated empirically, then we can deduce the degree to which the progenitor details can vary before they impact the accuracy with which the PNLF serves as a distance indicator.

First, I will illustrate that the PNLF provides valid extragalactic distances. I then consider that constraint in deducing properties of the PN progenitor populations.

\section{The PNLF Method Works}

Distances to all galaxies are suspect. Distances to even the closest galaxies, such as the Magellanic Clouds, are uncertain at the $5 \%$ to $10 \%$ level despite the opportunity to apply a multitude of distance measuring techniques. We never know the distance to a galaxy a priori, and so no test of any distance measuring method is perfect. Yet, verification via intercomparisons of methods is a crucial step toward building confidence that a particular technique works, and of equal importance, in estimating the level of accuracy that the method attains.

For the PNLF method, we have completed the following tests:

1. Tests within a single galaxy cluster applied to E/S0 galaxies (test for repeatability in old populations; Ciardullo et al. (1989))

2. Tests within a single galaxy group applied to spiral/elliptical pairs (test for Hubble type dependence; Ciardullo, Jacoby, \& Harris (1991); Jacoby, Ciardullo, \& Harris (1996))

3 . Tests across a range of metallicity (test for $\mathrm{O} / \mathrm{H}$ dependence; Ciardullo \& Jacoby (1992)) 
4. Tests against other methods (test for systematic effects; Ciardullo, Jacoby, \& Tonry (1993); Jacoby (1995), Feldmeier, Ciardullo, \& Jacoby (1997))

The last test is the most important since passing this one while failing any other suggests the unlikely presence of a cosmic conspiracy. Detailed tests were performed against the Surface Brightness Fluctuation method (Ciardullo, Jacoby, \& Tonry 1993), and less complete tests against the Globular Cluster Luminosity Function, $\mathrm{D}_{n}-\sigma$, and SN Ia methods were presented by Jacoby (1995).

The overwhelming acceptance of Cepheid distances and general skepticism of all other techniques, however, demands that the PNLF method be re-checked against this classic technique (see Cepheid reviews by Madore \& Freedman 1991; Jacoby et al. 1992).

\subsection{COMPARISON WITH CEPHEIDS}

PNLF distances now exist for 34 galaxies (see Table 1). Of these galaxies, 16 are late-type and therefore are suitable for comparison with Cepheid distances. Distances in Table 1 must be increased by 0.06 mag to be placed on the recent M31 Cepheid scale of Freedman \& Madore (1990). A metallicity correction (Ciardullo \& Jacoby 1992) has been applied to NGC 5253 because it has SMC-like abundances.

Feldmeier, Ciardullo, \& Jacoby (1997; FCJ) describe how the PNLF method can be extended to spiral galaxies. (See Jacoby et al. (1992) for a general description of the PNLF method.) Historically, the PNLF technique had been applied mostly to early-type galaxies to avoid confusion and contamination with HII regions and the effects of dust. Extending the method to spirals is crucial in order to compare the PNLF distances with Cepheids. Using the on-band/off-band method of [OIII] $\lambda 5007$ imaging, FCJ require that a PN candidate in a spiral must appear stellar, be present in the on-band [OIII] frame, but absent in each of the off-band, $\mathrm{H} \alpha$, and R-band frames. Due to the generally bright background from HII regions and young stars, most PN are found far from any spiral arms.

These criteria could potentially bias identifications against finding PN derived from very young stars to produce a more uniform set of PN than might otherwise be expected for observations in late-type galaxies. Effectively, the selection works to enhance the reliability of the technique.

This revised PNLF method was applied by FCJ to M51, M96, and M101, and by Soffner et al. (1996) to NGC 300. Of these, only M51 lacks a Cepheid distance for comparison. With prior PNLF distances to M31, M81, LMC, and NGC 5253, there are 7 Cepheid calibrators for the PNLF. In addition, there exist 4 indirect test points where Cepheid distances have 


\begin{tabular}{|c|c|c|c|c|}
\hline Galaxy & Type & $\mathbf{N r} \cdot \mathbf{P N}$ & $(\mathbf{m}-\mathbf{M})_{0}$ & Reference \\
\hline \multicolumn{5}{|c|}{ Local Group } \\
\hline LMC & $\mathrm{SBm}$ & 42 & $18.44 \pm 0.18$ & Jacoby, Walker, \& Ciardullo (1990) \\
\hline SMC & $\operatorname{Im}$ & 8 & $19.09 \pm 0.29$ & Jacoby, Walker, \& Ciardullo (1990) \\
\hline 185 & $\mathrm{dE} 3 \mathrm{p}$ & 4 & $\ldots$ & Ciardullo et al. (1989) \\
\hline 205 & S0/E5p & 12 & $24.68 \pm 0.35$ & Ciardullo et al. (1989) \\
\hline 221 & $\mathrm{E} 2$ & 9 & $24.58 \pm 0.60$ & Ciardullo et al. (1989) \\
\hline 224 & $\mathrm{Sb}$ & 104 & $24.26 \pm 0.04$ & Ciardullo et al. (1989) \\
\hline \multicolumn{5}{|c|}{ NGC 1023 Group } \\
\hline 891 & $\mathrm{Sb}$ & 34 & $29.97 \pm 0.16$ & Ciardullo, Jacoby, \& Harris (1991) \\
\hline 1023 & SB0 & 97 & $29.97 \pm 0.14$ & Ciardullo, Jacoby, \& Harris (1991) \\
\hline \multicolumn{5}{|c|}{ Fornax Cluster } \\
\hline 1316 & S0p & 58 & $31.13 \pm 0.07$ & McMillan, Ciardullo, \& Jacoby (1993) \\
\hline 1399 & $\mathrm{E} 1$ & 37 & $31.17 \pm 0.08$ & McMillan, Ciardullo, \& Jacoby (1993) \\
\hline 1404 & $\mathrm{E} 2$ & 19 & $31.15 \pm 0.10$ & McMillan, Ciardullo, \& Jacoby (1993) \\
\hline \multicolumn{5}{|c|}{ Leo I Group } \\
\hline 3377 & E6 & 22 & $30.07 \pm 0.17$ & Ciardullo, Jacoby, \& Ford (1989) \\
\hline 3379 & E0 & 45 & $29.96 \pm 0.16$ & Ciardullo, Jacoby, \& Ford (1989) \\
\hline 3384 & SB0 & 43 & $30.03 \pm 0.16$ & Ciardullo, Jacoby, \& Ford (1989) \\
\hline 3368 & Sab & 25 & $29.85 \pm 0.15$ & Feldmeier, Ciardullo, \& Jacoby (1997) \\
\hline \multicolumn{5}{|c|}{ Virgo Cluster } \\
\hline 4374 & E1 & 37 & $30.98 \pm 0.18$ & Jacoby, Ciardullo, \& Ford (1990) \\
\hline 4382 & So & 59 & $30.79 \pm 0.17$ & Jacoby, Ciardullo, \& Ford (1990) \\
\hline 4406 & S0/E3 & 59 & $30.98 \pm 0.17$ & Jacoby, Ciardullo, \& Ford (1990) \\
\hline 4472 & E1/S0 & 26 & $30.71 \pm 0.19$ & Jacoby, Ciardullo, \& Ford (1990) \\
\hline 4486 & E0 & 201 & $30.73 \pm 0.19$ & Jacoby (1996) \\
\hline 4649 & So & 16 & $30.76 \pm 0.19$ & Jacoby, Ciardullo, \& Ford (1990) \\
\hline \multicolumn{5}{|c|}{ Coma I Group } \\
\hline 4278 & E1 & 23 & $30.04 \pm 0.18$ & Jacoby, Ciardullo, \& Harris (1996) \\
\hline 4494 & E1 & 101 & $30.54 \pm 0.14$ & Jacoby, Ciardullo, \& Harris (1996) \\
\hline 4565 & $\mathrm{Sb}$ & 17 & $30.12 \pm 0.17$ & Jacoby, Ciardullo, \& Harris (1996) \\
\hline \multicolumn{5}{|c|}{ NGC 5128 Group } \\
\hline 5102 & Sop & 19 & $27.47 \pm 0.22$ & McMillan, Ciardullo, and Jacoby (1994) \\
\hline 5128 & S0p & 224 & $27.73 \pm 0.04$ & Hui et al. (1993) \\
\hline 5253 & Amorph & 16 & $27.80 \pm 0.29$ & Phillips et al. (1992) \\
\hline \multicolumn{5}{|l|}{ Other } \\
\hline Bulge & Sbc & 22 & $14.54 \pm 0.20$ & Pottasch (1990) \\
\hline 300 & $\mathrm{Sc}$ & 10 & $26.78 \pm 0.40$ & Soffner et al. (1996) \\
\hline 3031 & $\mathrm{Sb}$ & 88 & $27.72 \pm 0.25$ & Jacoby et al. (1989) \\
\hline 3109 & $\mathrm{Sm}$ & 7 & $26.03 \pm 0.30$ & Richer \& McCall (1992) \\
\hline 3115 & So & 52 & $30.11 \pm 0.20$ & Ciardullo, Jacoby, \& Tonry (1993) \\
\hline 4594 & $\mathrm{Sa}$ & 204 & $29.76 \pm 0.13$ & Ford et al. (1996) \\
\hline $5194 / 5$ & $\mathrm{Sbc} / \mathrm{SB} 0$ & 38 & $29.56 \pm 0.15$ & Feldmeier, Ciardullo, \& Jacoby (1997) \\
\hline 5457 & Sc & 27 & $29.36 \pm 0.15$ & Feldmeier, Ciardullo, \& Jacoby (1997) \\
\hline
\end{tabular}


been measured to a spiral in the same group as a PNLF E/S0 galaxy (Virgo cluster, Fornax cluster, Leo Group, NGC 1023 cluster). These latter points provide additional confidence in the comparison, but cannot be used to quantify the accuracy of the method because there is no way to be sure that two different galaxies have the same distance, even if they appear to be in the same group.

Figure 1 illustrates the results of the Cepheid comparison. All distances are placed on a scale using M31 (770 kpc from Freedman \& Madore (1990); $\mathrm{E}(\mathrm{B}-\mathrm{V})=0.08$ from Burstein \& Heiles (1984)) as the sole calibrator. The upper panel shows excellent agreement between the PNLF and Cepheid distances (both direct and indirect). The lower panel, showing the errors in the distances, illustrates that all tests fall within $1 \sigma$ of zero error, except for M96. With M96 in the statistics, the RMS error is $7.7 \%$; with M96 excluded, the RMS error is $4.5 \%$. There is no reason to exclude M96 a priori, and its deviation of $17 \%$ (twice that of any other galaxy) is expected to occur $10 \%$ of the time if the errors distribute randomly.

The Cepheid distance to M96 (Tanvir et al. 1995) is based on 7 Cepheids. Madore \& Freedman (1991), though, show that a distance accurate to $10 \%$ requires $\sim 25$ Cepheids to map out the finite width of the PL relation in the $\mathrm{V}$ and I bands. With 7 Cepheids, the quoted uncertainty of $8 \%$ is probably $\sim 2 \times$ too small. Evidence that the Tanvir distance is too large comes from the distance to M95 (Graham et al. 1996) based on 45 Cepheids that falls within $5 \%$ of the PNLF distance to M96.

\subsection{TESTS INTERNAL TO GROUPS AND CLUSTERS}

Even if the PNLF distances reproduce the Cepheid distances, a Hubble type dependence could persist to induce a systematic error when the PNLF is applied to early-type galaxies. For example, if stars in early-type galaxies are older than stars in spirals, as one might suppose, then the E/S0 central star masses and their luminosities will be smaller than their spiral counterparts. Consequently, the [OIII] luminosities for $\mathrm{PN}$ in $\mathrm{E} / \mathrm{S} 0$ galaxies will be fainter and we would overestimate their distances.

Figures 2 and 3 illustrate the tests for a Hubble type dependency. Jacoby, Ciardullo, \& Harris (1996), Jacoby (1996), and FCJ present additional figures showing the spiral/elliptical agreement for the Coma I and Leo Groups. Figure 2 shows that the PNLF distances to NGC 891 (Sb) and 1023 (SB0) are identical, while the Cepheid distance to the companion spiral, NGC 925, is also consistent (Silbermann et al. 1996).

The Virgo comparison shown in Figure 3 is more intriguing because of the large number (11) of galaxies. There are no PNLF distances to the spirals, but Figure 1 suggests that the Cepheid distances should serve as 
PNLF DISTANCES TO GALAXIES

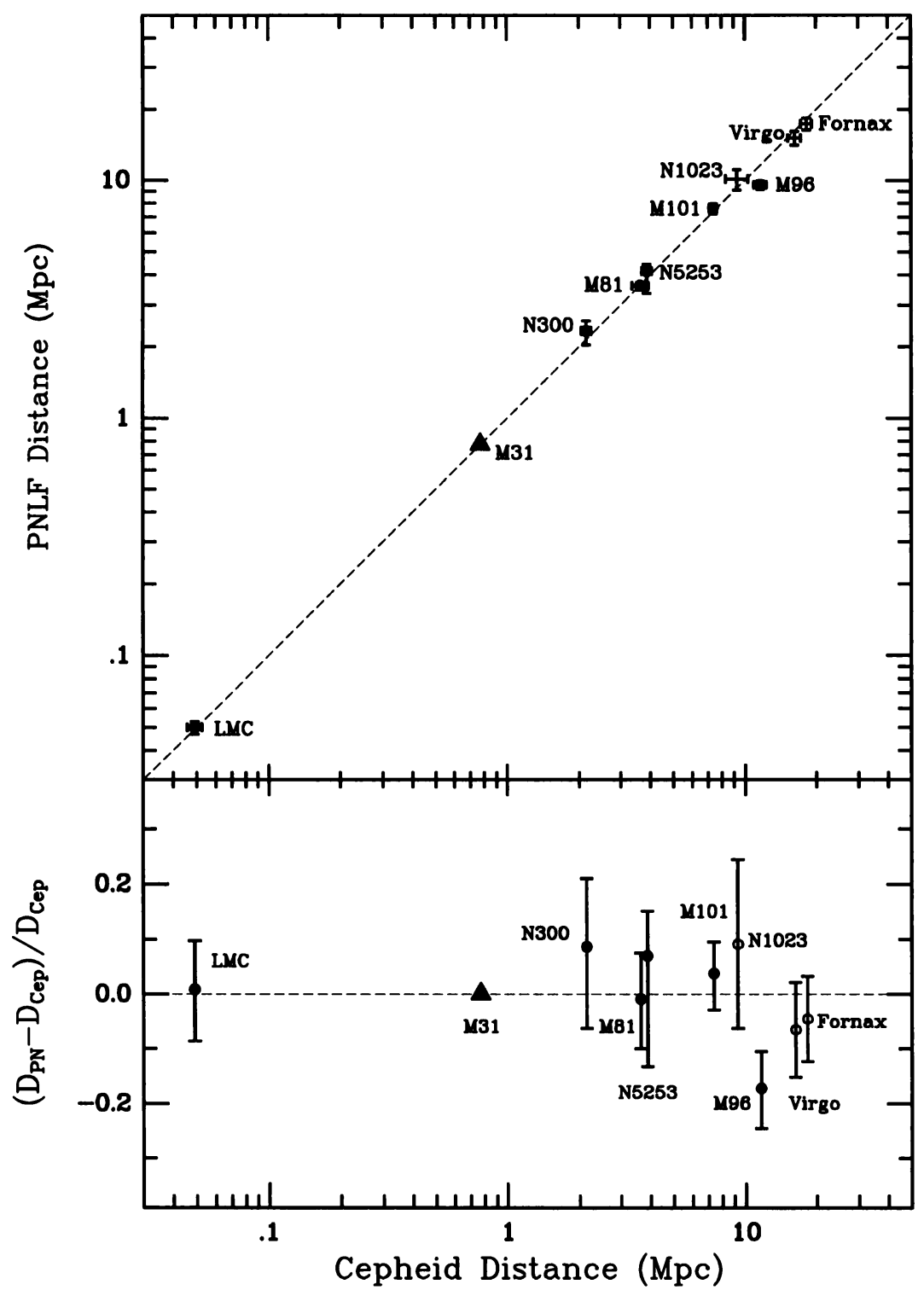

Figure 1. A comparison between PNLF and Cepheid distances. Solid circles represent direct galaxy comparisons; the solid triangle is the calibrator galaxy, M31, which, by definition, falls exactly on the dashed 1:1 line. The points labeled N1023, Virgo, and Fornax represent comparisons between elliptical (PNLF) and spiral (Cepheid) galaxies within the same cluster. The lower panel clarifies the level of disagreement by plotting the relative differences in distances. 


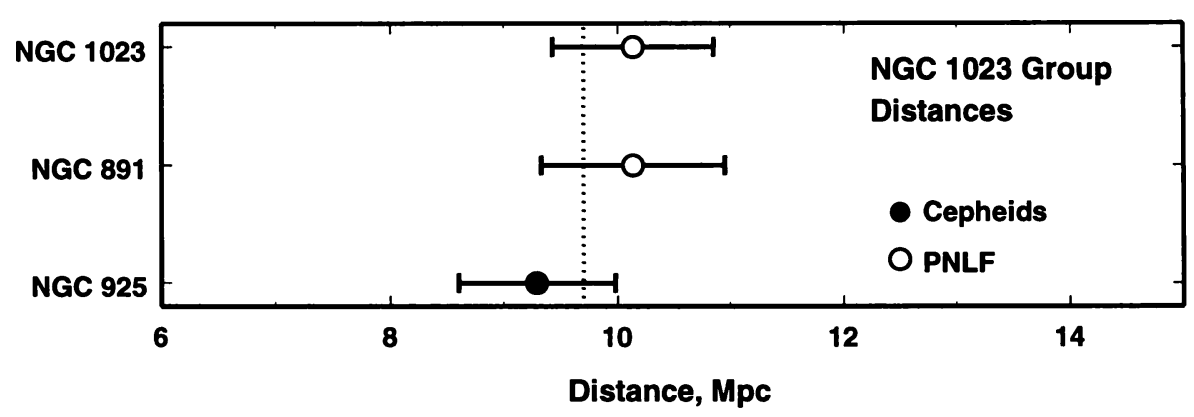

Figure 2. A comparison between PNLF and Cepheid distances in the NGC 1023 Group. A vertical line at $9.8 \mathrm{Mpc}$ has been drawn to suggest a possible average cluster distance.

adequate surrogates. The consistency among 10 of the 11 distances is striking and strongly suggests that the distance to Virgo is about $16 \mathrm{Mpc}$. Only one galaxy, NGC 4639 , falls outside the range 14-17 Mpc. Caution is essential in deciphering what these distances mean because it is not clear which galaxies are true members of the complex Virgo cluster.

Tammann \& Federspiel (1996) interpret the distances to NGC 4321 and NGC 4639 as representative of Virgo despite their 9 Mpc line of sight separation, and that NGC 4496A, NGC 4536, and NGC 4571 are foreground members of a subcluster. Curiously, though, the subcluster and NGC 4321 (M100) all fall at the PNLF distance of the $6 \mathrm{E} / \mathrm{S} 0$ galaxies. This agreement may be fortuitous, but Occam's razor suggests that NGC 4639 falls behind Virgo as defined by the remaining 10 galaxies.

\section{Derived Properties of the PN Progenitors}

Method intercomparisons indicate that the PNLF technique is accurate to $\sim 8 \%$ (Ciardullo, Jacoby, \& Tonry 1993; FCJ). Also, PNLF tests internal to specific clusters demonstrate excellent consistency $(\sim 3 \%)$. Thus, for the PNLF to yield absolute distances accurate to $<8 \%$, the maximum luminosities of PN in various populations must be similar to $<16 \%$, and thus, the populations cannot be too dissimilar.

\subsection{PROGENITOR AGES}

Since the PNLF shape depends mildly on progenitor age (Méndez et al. 1993; Stanghellini 1995), those ages cannot be extremely different. More importantly, while the PNLF cutoff luminosity is mildly sensitive to age, it becomes very sensitive for populations younger than $\sim 3 \mathrm{Gyr}$ (but see below). 


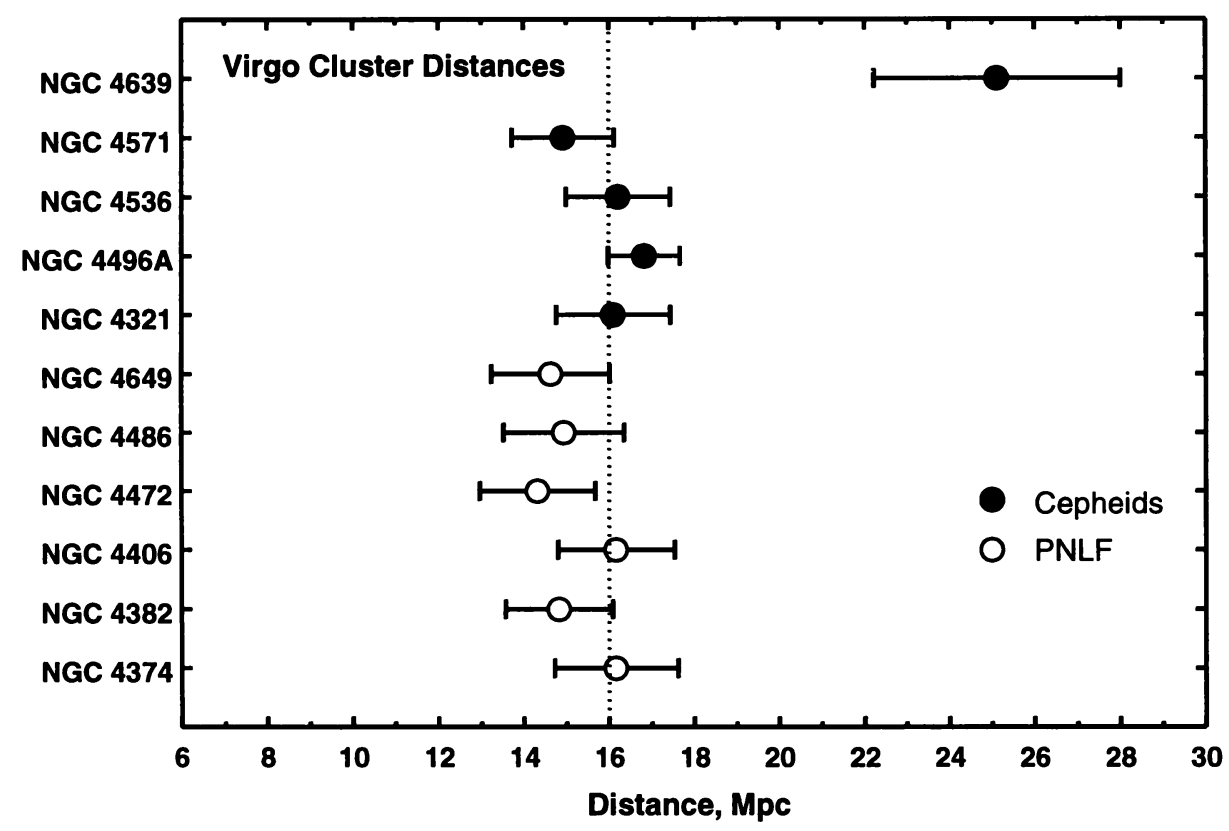

Figure 3. A comparison between the 6 PNLF distances to Virgo E/S0 galaxies and the 5 Cepheid spiral distances (Pierce et al. 1994; summary in Tammann \& Federspiel 1996). One clearly lies beyond the other 10 galaxies. A vertical line at $16 \mathrm{Mpc}$ is drawn to illustrate a possible choice for the average cluster distance.

We can probe the effects of population age in the following manner. We adopt the Weidemann (1987) initial-to-final mass relation and the Paczynski (1971) core mass - luminosity relation. Further, Dopita et al. (1992) estimated that the maximum flux emitted in [OIII] $\lambda 5007$ relative to the core luminosity is $\sim 15 \%$. We can now derive an approximate relation between progenitor age and maximum [OIII] $\lambda 5007$ luminosity (see Figure 4). The brightest PN progenitors all must be between 3 and 10 Gyrs for the PNLF method to yield reliable distances within 0.16 mag.

While a very old $(>12 \mathrm{Gyr})$ population yields distances that are slightly too large, stars younger than $\sim 3$ Gyrs yield distances that are very much too small. Thus, the presence of many very young stars seems unlikely. Kaler \& Jacoby (1991), though, showed that young progenitors do not produce bright PN. They attributed this counter-intuitive result to the effects of dredge-up whereby nitrogen is greatly enhanced in the Type I PN derived from the more massive progenitors. Nitrogen competes with oxygen to cool the nebula and depresses the [OIII] flux. In addition, the optical thickness 


\section{G. Jacoby}

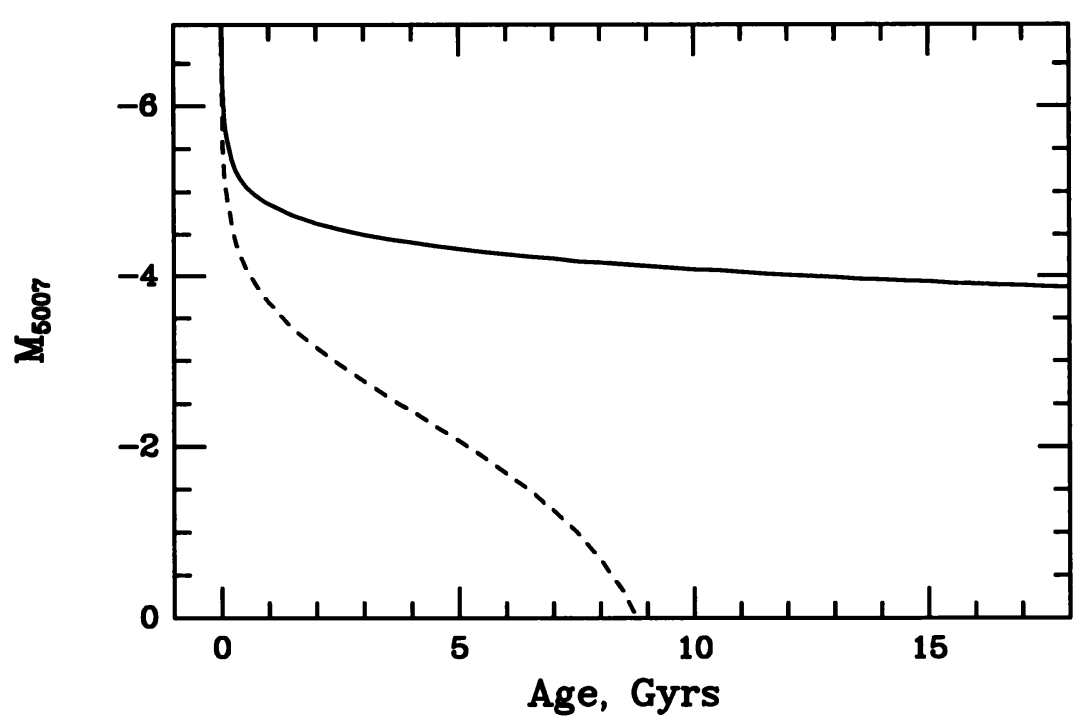

Figure 4. The effect of age on the maximum [OIII] luminosity. The simple approximation (solid line) falls $\sim 0.3 \mathrm{mag}$ too faint, suggesting that the initial-to-final mass relation is slightly too steep, that the core mass - luminosity relation is too faint, or that nebulae are $>15 \%$ efficient at converting UV to [OIII] flux. The detailed model (Jacoby 1989; Figure 5) yield luminosities (dashed line) faint by $2 \mathrm{mag}$ unless the PN progenitors are very young or the Weidemann function is too steep. Stars older than 15 Gyrs might be excluded from the diagram because the time predicted for their low mass central stars to heat to photoionization temperatures exceeds the nebula dissipation lifetime.

of Type I PN may be smaller than in PN derived from intermediate age progenitors (Méndez \& Soffner 1996), again reducing the [OIII] luminosity. Two additional processes come into play: Type I PN have rapidly evolving central stars that are unlikely to be observed when at their brightest; also, the nebulae tend to contain considerable dust.

Are stars in all galaxies younger than 10 Gyrs? This seems unreasonable for ellipticals. The PNLF reliability, though, argues that the stars producing bright PN must be younger if the initial-to-final mass relation is as steep as adopted, and if the central star evolutionary time scales are correct. With these assumptions and the production rates for bright PN, $\sim 25 \%$ of the stars derive from intermediate age progenitors. This estimate is consistent with the broadband colors and spectral indices of ellipticals (Gonzalez 1993) where intermediate ages are deduced for many of the galaxies observed in the PNLF studies. Also, the accuracy of the PNLF method suggests that a significant fraction of stars in the disks of spiral galaxies have ages similar to a significant fraction of stars in ellipticals.

The simple model in Figure 4 neglects various effects such as temperature and luminosity evolution of the central stars and expansion of the 
nebulae that are considered in Jacoby's (1989) models (see Figure 5 of Jacoby) When combined with a Weidemann initial-to-final mass relation, the bright PN are $\sim 2$ mag too faint unless the ages of many stars are $<1 \mathrm{Gyr}$. Key issues that may be leading to the low luminosity are:

1. Overestimate of the nebula expansion velocity. Jacoby (1989) adopted $20 \mathrm{~km} / \mathrm{s}$ whereas Gussie \& Taylor (1994) find that velocity increases with nebula size; small $\mathrm{PN}$ have velocities near $15 \mathrm{~km} / \mathrm{s}$. This represents a known deficiency of the Jacoby models.

2. Possible underestimate of the evolutionary rates for low mass central stars $\left(\mathrm{M}<0.6 M_{\odot}\right)$. This item is speculative.

3. Possible overestimate in the slope of the initial-to-final mass relation.

Additional detailed modeling of the PNLF is needed to assess the relative importance of these items. The most intriguing is that the PNLF results are revealing our ignorance about the initial-to-final mass relation where it is most uncertain: at the lower masses. Another wildcard is the effect of binary star evolution (see paper by Yungelson in this volume).

\subsection{EFFECTS OF DUST}

Dust in the regions of the galaxies studied cannot be affecting the apparent brightnesses of the most luminous PN. FCJ show that if the PN population has a scale height several times larger than the dust scale height, as in our galaxy, then the brightest observed PN are unaffected by dust. Distances to dusty galaxies are affected $<5 \%$ (see Figure 4 of FCJ).

Similarly, dust present within nebulae cannot affect the brightest nebulae by $>0.16 \mathrm{mag}$. This would seem to be a fortuitous situation. A simpler explanation is that dust around the hot central stars powering the brightest nebulae is destroyed or dispersed.

\subsection{EVOLUTIONARY TIME SCALES}

The PNLF shape is a function of a variety of processes (Jacoby 1989). Among these are the heating and cooling times for PN central stars (e.g., Schönberner 1981), white dwarf mass distributions (e.g., McMahon 1989), and mass loss along the PAGB tracks (Vassiliadis \& Wood 1994). None of the adopted functionalities for these parameters can be very wrong or the predicted PNLF shape would differ significantly from the observed shape. Despite concerns about evolutionary rates of low mass stars expressed in $\S 3.1$, the bright end shape is governed primarily by central stars at $0.65 M_{\odot}$.

If the true evolutionary time scales were faster than assumed, there would be too few bright PN and the PNLF would truncate more rapidly and at a lower luminosity. Conversely, slower time scales produce a brighter, 


\section{G. Jacoby}

shallower cutoff. Further investigation is needed to estimate how large the time scale discrepancy can be before PNLF models deviate significantly from the observed shapes.

\subsection{METALLICITY}

Dopita et al. (1992) posed the theoretical arguments for insensitivity of the PNLF to metallicity. Ciardullo \& Jacoby (1992) provided a limited test of those arguments. The effect of metallicity on the nebula is countered by the metallicity effect on the central star. Fewer "metals" (actually, $[\mathrm{O} / \mathrm{H}]$ ) in the nebula means fewer atoms to emit $\lambda 5007$ photons, yielding a fainter nebula. Conversely, a central star derived from a low metallicity (actually, $[\mathrm{Fe} / \mathrm{H}]$ ) star will be more massive and more luminous than one derived from a high metallicity star. The more luminous central star partially compensates for the lower luminosity potential of the nebula. This argument has not been thoroughly tested, but must be correct to within the reliability limits of the PNLF method (0.16 mag). The Dopita et al. (1992) formulation implies that the metallicities of most of these galaxies fall within $2 \times$ solar.

\section{Conclusions}

This paper summarizes the tests and arguments that demonstrate $8 \%$ reliability for the PNLF extragalactic distance technique. For the PNLF method to work as well as it appears to, certain aspects of PN formation and evolution must be constrained. Among these are:

- Theoretical evolutionary time scales for $0.60-0.65 \mathrm{M}_{\odot}$ central stars must be approximately correct for models to match the observed PNLF shapes.

- Progenitors of the brightest PN must have ages smaller than 10 Gyrs. Possibly, very young progenitors do not produce bright PN because of nitrogen pollution or dust in the nebula, or perhaps the nebulae they produce are optically thin.

- Dust within the brightest nebulae is minimal, perhaps because the dust is dispersed or dissociated by the time the nebula becomes bright.

- Metallicity differences among populations cannot have a significant effect on the luminosity of the brightest PNe. This implies a correlation between $\mathrm{Fe} / \mathrm{H}$ and $\mathrm{O} / \mathrm{H}$.

- There is discordance between the models of Jacoby (1989) and the luminosities predicted under the Weidemann (1987) initial-to-final mass relation. Several areas for future investigation were noted such as the effects of slower nebular expansion velocity, faster evolution of low mass central stars, and shallow initial-to-final mass functions. 


\section{PNLF DISTANCES TO GALAXIES}

\section{References}

Burstein, D. \& Heiles, C. (1984) Ap.J.Suppl. 54, 33.

Ciardullo, R. \& Jacoby, G. H. (1992) Ap.J. 388, 268.

Ciardullo, R., Jacoby, G. H., Ford, H. C., \& Neill, J. D. (1989) Ap.J. 339, 53.

Ciardullo, R., Jacoby, G. H., \& Harris, W. E. (1991) Ap.J. 383, 487

Ciardullo, R., Jacoby, G. H., \& Tonry, J. L. (1993) Ap.J. 419, 479.

Dopita, M. A., Jacoby, G. H., \& Vassiliadis, E. (1992) Ap.J. 389, 27.

Feldmeier, J., Ciardullo, R., \& Jacoby, G. H. (1997) Ap.J., in press.

Ford, H. C., Hui, X., Ciardullo, R., Jacoby, G. H., \& Freeman, K. C. (1996) Ap.J. 458, 455.

Freedman, W. L. \& Madore, B. F. (1990) Ap.J. 365, 186.

Gonzalez 1993, PhD Thesis, Univ. California at Santa Cruz

Graham, J. A., Phelps, R. L., Freedman, W. L., Saha, A., Stetson, P. B., Madore, B. F., Silbermann, N. A., Sakai, S., Kennicutt, R. C., Harding, P., Turner, A., Mould, J. R., Ferrarese, L., Ford, H. C., Hoessel, J. G., Han, M., Huchra, J. P., Hughes, S. M., Illingworth, G. D., \& Kelson, D. D. (1996) Ap.J., in press.

Gussie, G. T., \& Taylor, A. R. (1994) P.A.S.P. 106500.

Hui, X., Ford, H. C., Ciardullo, R., \& Jacoby, G. H. (1993) Ap.J. 414463.

Jacoby, G. H. (1989) Ap.J. 339, 39.

Jacoby, G. H. (1995) in Science With the VLT, eds. J. R. Walsh \& I. J. Danziger, 267.

Jacoby, G. H. (1996) in Proceedings of the Space Telescope Science Institute Symposium on The Extragalactic Distance Scale, eds. M. Livio \& M. Donahue, in press.

Jacoby, G. H., Branch, D., Ciardullo, R., Davies, R. L., Harris, W. E., Pierce, M. J., Pritchet, C. J., Tonry, J. L., \& Welch, D. L. (1992) PASP 104, 599.

Jacoby, G. H., Ciardullo, R., \& Harris, W. E. (1996) Ap.J. 462, 1.

Jacoby, G. H., Walker, A. R., \& Ciardullo, R. (1990) Ap.J. 365, 471.

Kaler, J. B. \& Jacoby, G. H. (1991) Ap.J. 382, 134.

Madore, B. F. \& Freedman, W. L. (1991) PASP, 103, 933.

Méndez \& Soffner (1996), in IAU Symposium 180, Planetary Nebulae, in press.

Méndez, R. H., Kudritzki, R. P., Ciardullo, R. P., \& Jacoby, G. H. (1993) Astr. Ap. 275 , 534.

McMahon, R. K. (1989) Ap.J. 336, 409.

McMillan, R., Ciardullo, R., \& Jacoby, G. H. (1993) Ap.J. 416, 62.

McMillan, R., Ciardullo, R., \& Jacoby, G. H. (1994) AJ 108, 1610.

Paczynski (1971) Acta.Astron. 21, 417.

Phillips, M. M., Jacoby, G. H., Walker, A. R., Tonry, J. L., \& Ciardullo, R. (1992) B.A.A.S. 180, 1304.

Pierce, M. J., Welch, D. L., McClure, R. D., van den Bergh, S., Racine, R., \& Stetson, P. B. (1994) Nature 371, 385.

Pottasch, S. R. (1990) Astr.Ap. 236, 231.

Richer, M. G., \& McCall, M. L. (1992) $A J$ 103, 54.

Schönberner, D. (1981) Astr.Ap. 103, 119. bibitem[ Silbermann, N. A., and 18 others (1996) Ap.J. 470, 1.

Soffner, T., Méndez, R. H., Jacoby, G. H., Ciardullo, R., Roth, M. M., \& Kudritzki, R. P. (1996) Astr.Ap. 306, 9.

Stanghellini, L. (1995) Ap.J. 452, 515.

Tammann, G. A. \& Federspiel, M. (1996) in Proceedings of the Space Telescope Science Institute Symposium on The Extragalactic Distance Scale, eds. M. Livio \& M. Donahue, in press.

Tanvir, N. R., Shanks, T., Ferguson, H. C., \& Robinson, D. R. T. (1995) Nature 377, 27.

Vassiliadis, E., \& Wood, P.R. (1994) Ap.J.Sup. 92, 125.

Weidemann, V. (1987) Astr.Ap. 188, 74. 